\title{
Human $\mathrm{B} 7-\mathrm{H} 3$ binds to Triggering receptor expressed on myeloid cells-like transcript 2 (TLT-2) and enhances $T$ cell responses
}

\author{
Masaaki Hashiguchi ${ }^{1,2^{*}}$, Yuka Inamochi ${ }^{1}$, Shoya Nagai ${ }^{1}$, Noriko Otsuki ${ }^{1}$, Jinhua Piao ${ }^{1}$, \\ Hiroko Kobori ${ }^{1}$, Yumiko Kanno ${ }^{2}$, Hidefumi Kojima ${ }^{2}$, Tetsuji Kobata ${ }^{2}$, Miyuki Azuma ${ }^{1}$ \\ ${ }^{1}$ Department of Molecular Immunology, Graduate School of Medical and Dental Sciences, Tokyo Medical and Dental University, \\ Tokyo, Japan; "Corresponding Author: mhashi@dokkyomed.ac.jp \\ ${ }^{2}$ Department of Immunology, School of Medicine, Dokkyo Medical University, Tochigi, Japan
}

Received 21 September 2011; revised 18 November 2011; accepted 8 December 2011

\section{ABSTRACT}

The B7 family member B7-H3 is broadly expressed in many tissue and tumor types. B7-H3 expression is induced on some immune cells; however, its immunological function remains controversial, because both immunoenhancing and immunoinhibitory effects have been reported in human and mouse systems. We have previously reported the following: 1) murine B7-H3 specifically bound to Triggering receptor expressed on myeloid cells (TREM)-like transcript 2 (TLT-2, TREML2), a member of the TREM family of receptors; and 2) the B7-H3:TLT-2 pathway up-regulated $\mathrm{T}$ cell responses. However, the expression and function of human TLT-2 has not yet been clarified. A recent study found no evidence to support the existence of an interacttion between human B7-H3 and TLT-2. In this study, we demonstrated that human B7-H3 binds to TLT-2 and augments T cell responses. Human and mouse B7-H3lg chimeric proteins crossinteracted with both human and mouse species of TLT-2-transduced cells. Human TLT-2 was expressed on freshly isolated, peripheral blood $B$ cells and monocytes, and subpopulations of $\mathrm{CD}^{+}$and $\mathrm{CD}^{+} \mathrm{T}$ cells. Human TLT-2 expression on $\mathrm{T}$ cells did not correlate with naïve or memory phenotypes and was diminished after culture, despite the presence of mitogenic stimuli. Constitutive TLT-2 expression on monocytes was also down-regulated after culture. Human B7-H3 transfectants augment IL-2 production from TLT-2-transduced T cell hybridomas, and IFN- $\gamma$ production from peripheral blood CD4 ${ }^{+}$and CD8 ${ }^{+}$ $T$ cells. The enhanced responses were inhibited by the addition of anti-TLT-2 mAbs, suggesting TLT-2-mediated costimulatory effect. Our results demonstrate the existence of a functional interaction between human B7-H3 and TLT-2, and the restricted expression of TLT-2 on $\mathrm{T}$ cells and monocytes.

Keywords: B7-H3; Co-Signal Molecule; T Cell; TLT-2

\section{INTRODUCTION}

A $\mathrm{T}$ cell response is triggered by interaction with antigen-presenting cells. This response is positively or negatively modulated by various co-signal molecules [1-3]. B7-H3 (CD276) is a member of the B7 family, which was originally identified as costimulatory molecules for $\mathrm{T}$ cell activation [4]. B7-H3 exists in 2 forms in humans - a majority possesses 4 immunoglobulin-like domains (4Ig), while a minority possesses $2 \mathrm{Ig}$ - but only in 1 form (2Ig) in mice [5-7]. No functional difference has been observed between the 2 forms of B7-H3 [5,8]. Human B7-H3 is induced on activated monocytes and dendritic cells (DCs) and has also been detected on airway and nasal epithelial cells, muscle cells, and synoviocytes during inflammatory conditions, as well as on many types of tumors [4,7,9-18]. Like the murine system, the immunological function of human B7-H3 remains controversial. Human B7-H3Ig fusion protein costimulated anti-CD3 $\mathrm{mAb}$-induced proliferation of $\mathrm{CD} 4^{+}$and $\mathrm{CD} 8^{+}$ $\mathrm{T}$ cells and selectively enhanced IFN- $\gamma$ production. Furthermore, stimulation with B7-H3-transfected tumors preferentially up-regulated the proliferation and IFN- $\gamma$ production of $\mathrm{CD}^{+}$T-cell responses in vitro [4,19]. RNAi knockdown of B7-H3 expression on fibroblastlike synoviocytes reduced the production of TNF- $\gamma$, IFN- $\gamma$ and IL-2 from cytokine-activated T cells [17]. These results suggest costimulatory roles for $\mathrm{B} 7-\mathrm{H} 3$ in $\mathrm{T}$ cellmediated immune responses. In contrast, other groups have 
demonstrated that both forms of human B7-H3Ig inhibited cellular proliferation and cytokine production (e.g., IFN- $\gamma$, IL-2, and IL-10) by $\mathrm{CD}^{+}, \mathrm{CD}^{+}$, and naïve/preactivated human $\mathrm{T}$ cells $[5,8]$. Knockdown of B7-H3 expression in muscle cells enhanced $\mathrm{CD} 8^{+} \mathrm{T}$ cell-specific lysis [18]. Expression of tumor-associated B7-H3 and its correlation with clinical status, pathological grade, and/or survival rate have been extensively studied. Studies examining the tumorigenic activity of B7-H3-including carcinomas of the prostate [14,20], kidney [21], lung [13], hypopharynx [22], colon [23], stomach [12] and pancreas [24] - revealed that tumor-associated B7-H3 correlated with higher tumor grade, lymph node- or distant-metastasis, fewer tumor-infiltrating lymphocytes (TILs), and/or poor survival. Only one report on pancreatic cancer has suggested a stimulatory role for B7-H3 in antitumor immune responses by demonstrating the correlation between tumor-associated B7-H3 and higher TILs and improved prognosis [25].

Triggering receptor expressed on myeloid cells (TREM) and TREM-like receptors are a structurally related family of proteins encoded by genes clustered on mouse chromosome $17 \mathrm{C}$ and human chromosome 6p21 [26,27]. They are expressed on a variety of innate immune cells of myeloid lineage, including neutrophils, macrophages, myeloid dendritic cells, microglia, osteoclasts, and platelets. Unlike other TREM family members, TREM-like transcript-2 (TLT-2, TREML2) is expressed on immune cells of lymphoid lineage (e.g., $\mathrm{T}$ and $\mathrm{B}$ cells) as well as myeloid cells; moreover, unlike TREM1 and TREM2, TLT-2 does not bind to adaptor molecules such as immunoreceptor tyrosine-based activation motif (ITAM)-containing DAP12 [27,28]. Expression of mouse TLT-2 on neutrophils and macrophages is up-regulated in response to inflammation; TLT-2 may contribute to innate cell activation and chemotaxis $[28,29]$. We reported previously that murine TLT-2 is a counter-receptor for mouse B7-H3, and the TLT-2:B7-H3 pathway enhances $\mathrm{T}$ cell responses in vitro and in vivo [30]. TLT-2 is constitutively expressed on $\mathrm{CD}^{+} \mathrm{T}$ cells and induced on $\mathrm{CD}^{+} \mathrm{T}$ cells after stimulation. Transduction of B7-H3 into B7-H3 $3^{\text {null-low }}$ tumor cells efficiently enhanced antitumor responses by enhancing effector $\mathrm{CD} 8^{+} \mathrm{T}$-cell function; blockade of the B7-H3:TLT-2 pathway accelerated the growth of tumors that express endogenous B7-H3 [31]. In tumor-bearing mice, TLT- 2 was preferentially expressed on $\mathrm{CD}^{+} \mathrm{T}$ cells in the regional lymph node, but was down-regulated in TILs. Recently, it was demonstrated that both forms of human B7-H3Ig did not bind to human TLT2, and mouse B7-H3Ig did not bind to mouse TLT-2 [8]. In this study, however, we demonstrate that human and mouse B7-H3Ig cross-interact with both human and mouse TLT-2 species, and that interactions of human TLT-2 with human B7-H3 additionally costimulate $\mathrm{T}$ cell activation.

\section{MATERIAL AND METHODS}

\subsection{Cell Lines}

Human T lymphoma cell lines (Jurkat, Molt4, PEER, and YT2C2), Burkitt's leukemia cell lines (Daudi, Raji, and Ramos), EBV-transformed B lymphoblastoid cell line (Nalm-6 and JY), myeloid and histiocytic leukemia cell lines (THP-1 and U937), and a pre-B cell lymphoma line (697) were used. All human cell lines, murine mastocytoma P815 cells, and T cell hybridoma DO11.10 cells were cultured in RPMI1640 media containing 10\% fetal bovine serum and antibiotics.

\subsection{Gene Transduction and Chimeric Fusion Proteins}

Full-length human 4IgB7-H3 (CD276) and human TLT-2 (TREML2) cDNA were isolated by RT-PCR from a renal adenocarcinoma cell line (ACHN) and peripheral blood $\mathrm{CD} 8^{+} \mathrm{T}$ cells, respectively, as described previously [32]. The cDNA was subcloned into the pMKITneo expression vector and the pMXs-IG and pMXs-neo retroviral vectors (kindly provided by Dr. T. Kitamura, University of Tokyo). In some experiments, both mouse and human TLT-2 were tagged with a FLAG sequence on $\mathrm{N}$-termini excised from the leader sequence by PCR amplification of the corresponding regions. The amplified cDNA was ligated onto the pMXs vectors containing human CD8A leader sequence. The gene-transduced cell lines were established as previously described [30]. Human B7-H3Ig (hB7-H3Ig) (consisting of human B7-H3 amino acids $1-458$ and human IgG1 $\mathrm{Fc}$ ) and mouse B7-H3Ig (mB7-H3Ig) were prepared as described previously [33].

\subsection{Generation of MAbs against Human B7-H3 and TLT-2}

Hybridomas against human B7-H3 (MIH39 and MIH42, both mouse IgG1, $\kappa$ ) or human TLT-2 (MIH56, mouse IgM, $\kappa$; MIH59, IgG1, $\kappa$; MIH60, IgG2b, $\kappa$ MIH61, IgG1, $\kappa$ ) were generated by fusing P3U1 myeloma and immunized splenocytes, as described previously [32]. An anti-mouse/human B7-H3 mAb (MIH32, rat IgG2a, $\kappa$ ) was generated as described previously [30]. Human B7-H3/P815 and human TLT-2/DO11.10 cells were used for immunization. mAbs were purified from the culture supernatant with a Protein G column, and their purity was verified by SDS-PAGE. FITC-conjugation and biotinylation were performed using a standard method.

\subsection{MAbs and Flow Cytometry}

mAbs against human CD3 (OKT3), CD4 (OKT4), CD8 (OKT8), CD14 (63D3), CD19 (HIB19), and CD45RA (HI100), and murine CD3 (145-2C11) were 
used. Isotype control Ig (mouse IgG1, MOPC21, SigmaAldrich) was used for the control staining. For biotinylated mAbs, phycoerythrin (PE)- or allophycocyanin (APC)-conjugated streptavidin was used for detection. All fluorochrome-conjugated or biotinylated mAbs and reagents were obtained from eBioscience or BD PharMingen, unless otherwise noted. Flow cytometry was performed using FACSCalibur (BD Biosciences) and FlowJo (TreeStar) software.

\subsection{Cell Preparation and T Cell Stimulation Assay}

Peripheral blood mononuclear cells (PBMCs) and $\mathrm{CD}^{+}$and $\mathrm{CD}^{+}$cells were isolated as described previously [32]. PBMCs were stimulated with $2 \mu \mathrm{g} / \mathrm{ml}$ phytohemagglutinin (PHA; Sigma-Aldrich) or $10 \mu \mathrm{g} / \mathrm{ml}$ LPS (Sigma-Aldrich) for 3 or 7 days, and subjected to flow cytometry analyses. Parental DO11.10 and TLT-2/DO11.10 cells (both $1 \times 10^{4} /$ well) and $\mathrm{CD}^{+}$and $\mathrm{CD} 8^{+} \mathrm{T}$ cells $(1 \times$ $10^{5} /$ well) were stimulated with the indicated density of mitomycin C-treated parental P815 or B7-H3/P815 cells in the presence of $1 \mu \mathrm{g} / \mathrm{ml}$ anti-CD3 $\mathrm{mAb}$ in 96-well flat-bottom plates for $24 \mathrm{~h}$ and 3 days, respectively. The production of IL- 2 or IFN- $\gamma$ in the culture supernatants was measured using Ready-Set-Go ELISA systems (eBioscience), as described previously [32]. In some experiments, isotype control Ig (mouse IgG1, MOPC21, SigmaAldrich) or anti-TLT-2 mAb (MIH59 and MIH60) was added at indicated concentrations at the start of the culture. The statistical differences were evaluated using MannWhitney $U$ test.

\section{RESULTS}

\subsection{Human B7-H3 Binds TLT-2}

To examine the binding of human B7-H3 to TLT-2, hTLT-2-transduced DO11.10 cells were generated, and graded doses of h4IgB7-H3Ig were added to parental DO11.10 and hTLT-2/DO11.10 cells before analysis by flow cytometry. Human B7-H3Ig bound to hTLT-2/DO11. 10 , but not to parental DO11.10, in a dose-dependent manner (Figure 1(a)). When hTLT-2/DO11.10 cells were

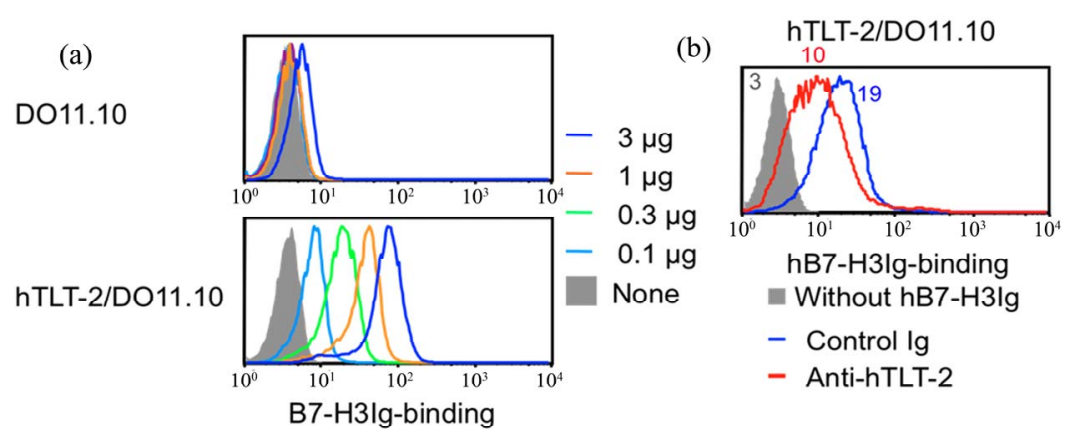

(c) D011.10

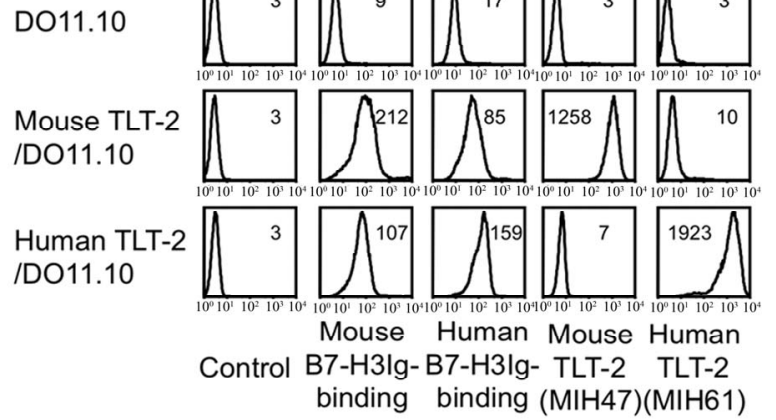

Figure 1. B7-H3 specifically binds to TLT-2 in humans. (a) Parental DO11.10 or hTLT-2/ DO11.10 cells were incubated with graded doses $(0.1,0.3,1$, or $3 \mu \mathrm{g})$ of biotinylated human $\mathrm{B} 7-\mathrm{H} 3 \mathrm{Ig}$, followed by streptavidin-PE. Representative histogram profiles are shown, with the control histograms nearest the ordinate (shaded); (b) Human TLT-2/ DO11.10 cells were preincubated with control mouse Ig (blue line) or anti-TLT-2 mAb (MIH56, red line) before staining with $1 \mu \mathrm{g}$ of B7-H3Ig as in (a). The control histogram without staining with B7-H3Ig is shown nearest the ordinate (shaded); (c) Parental DO11.10, mouse or human TLT-2-transduced DO11.10 cells were incubated with $1 \mu \mathrm{g}$ of biotinylated control human IgG1, mouse B7-H3Ig, or human B7-H3Ig, or stained with biotinylated anti-murine (MIH47) or human (MIH61) TLT-2 mAb, followed by streptavidin-PE. Representative histogram profiles are shown. 
pretreated with anti-hTLT-2 mAb (MIH56) prior to incubation with h4IgB7-H3Ig, the binding of hB7-H3Ig to cell surface hTLT-2 was partially inhibited (Figure 1(b)). To examine the cross-interaction between species, the binding of m2IgB7-H3 and h4IgB7-H3Ig onto mouse or human TLT-2/DO11.10 cells was examined. Mouse 2IgB7H3Ig bound to both mouse and human TLT-2/DO11.10, but not to parental DO11.10 (Figure 1(c)). Similarly, human $4 \operatorname{IgB} 7-\mathrm{H} 3 \operatorname{Ig}$ bound to both human and mouse TLT-2/DO11.10, but not to parental DO11.10. Biotinylated control human IgG did not react with any TLT-2transduced cells. Anti-mouse TLT-2 mAb (MIH47) specifically bound to mouse TLT-2, but not to human TLT-2; anti-human TLT-2 mAb (MIH61) specifically bound to human TLT-2, but not to mouse TLT-2 (Figure 1). These results indicate that both human and mouse B7-H3 cross-interacted between mouse and human TLT-2 species, despite the observation that the specificities of mAbs against their respective species-specific TLT-2 forms were maintained. Because the extracellular domains of mouse and human TLT- 2 share a $52 \%$ homology at the amino acid level, the latter result was predictable.

\subsection{TLT-2 Expression}

Six hybridomas producing mAbs against human TLT-2 (MIH56-MIH61) were generated. All these mAbs specifically reacted with human TLT-2-transduced cell lines (DO11.10, J558L, and 2B4), but not with the parental cells (Figure 1(c) and data not shown). MIH61 showed the highest relative reactivity to cell surface TLT-2; therefore, MIH61 was used in subsequent flow cytometric analyses. Analysis of freshly isolated peripheral blood lymphocytes identified a subpopulation of both $\mathrm{CD} 4^{+}$ and $\mathrm{CD} 8^{+} \mathrm{T}$ cells that constitutively expressed TLT-2; a comparative analysis of CD45RA ${ }^{+}$and CD45RA ${ }^{-}$fractions did not show preferential expression in the naïve or memory phenotype (Figure 2(a)). By comparison, $\mathrm{CD} 19^{+}$ $\mathrm{B}$ cells and $\mathrm{CD} 14^{+}$monocytes expressed substantially higher levels of TLT-2. PBMCs were stimulated with either PHA for T cells or LPS for B cells and monocytes; TLT-2 expression on days 3 and 7 after stimulation was examined. Cell surface TLT- 2 expression on $\mathrm{CD}^{+}$and $\mathrm{CD}^{+} \mathrm{T}$ cells was gradually down-regulated even in the medium-alone culture and mostly diminished on day 7 . TLT-2 expression on $\mathrm{CD} 19^{+}$B cells did not clearly change, but LPS stimulation appeared to induce slightly decreased TLT- 2 expression. TLT- 2 expression on CD14 ${ }^{+}$ monocytes was also down-regulated in culture with or without LPS, although LPS stimulation maintained relatively constant TLT- 2 expression levels at day 3 . These results indicate that human TLT-2 is constitutively expressed on freshly isolated PBMCs that include $\mathrm{CD}^{+}$ and $\mathrm{CD} 8^{+} \mathrm{T}$ cells, $\mathrm{B}$ cells, and monocytes; however,

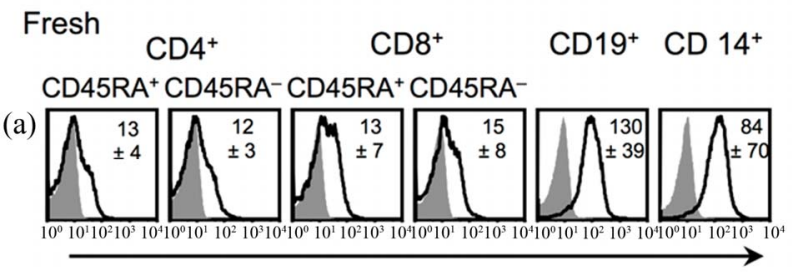

hTLT-2
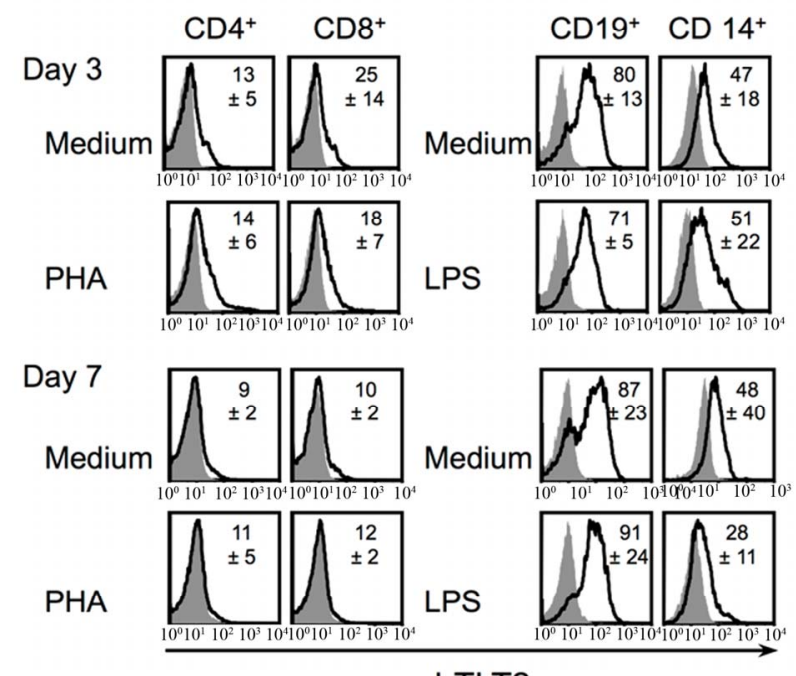

hTLT2
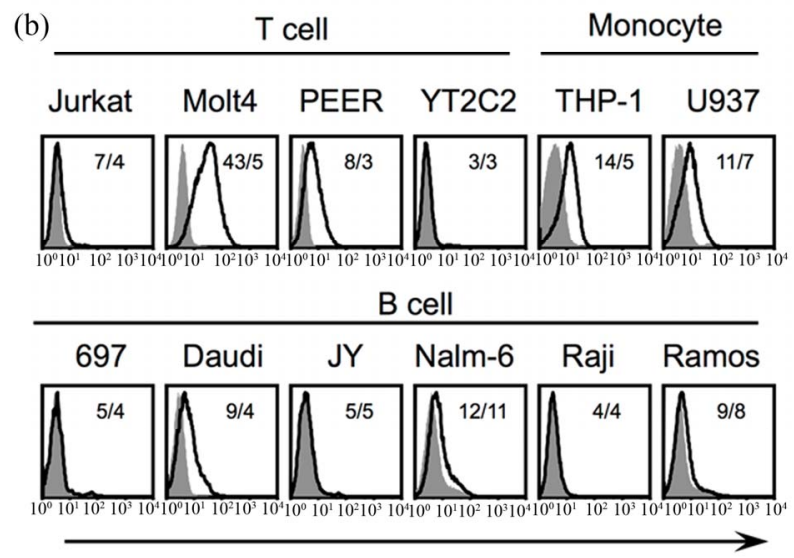

hTLT-2

Figure 2. TLT-2 expression; (a) Freshly isolated PBMCs from healthy donors or activated PBMCs stimulated with $2 \mu \mathrm{g} / \mathrm{ml}$ PHA or $10 \mu \mathrm{g} / \mathrm{ml}$ LPS for T cells or B cells and mono- cytes, respectively, for 3 and 7 days were stained with FITC-antiCD45RA and PE-anti-CD4 or PE-anti-CD8, or FITC-antiCD14 and PE-anti-CD19 mAbs in combination with biotinylated anti-TLT-2 mAb, followed by streptavidin-APC. An electronic gate was set on an indicated maker of lymphocytes; TLT-2 expression is shown as histogram profiles; (b) $\mathrm{T}$ cell lines (Jurkat, Molt4, PEER, and YT2C2), B cell lines (697, Daudi, JY, Nalm-6, Raji, and Ramos), and monocyte lines (THP-1 and U937) were stained with biotinylated anti-TLT-2 $\mathrm{mAb}$ (MIH61), followed by streptavidin-PE and analyzed by flow cytometry. Representative histogram profiles are shown with the control histograms nearest the ordinate (shaded). 
TLT-2 expression on T cells and monocytes was downregulated in culture with or without specific stimuli. Our results suggest that cell surface TLT-2 expression was not stable and was easily down-regulated.

TLT-2 expression on human cell lines was variable, with substantial expression observed in several cell lines (Molt4, PEER, THP-1, and U937) (Figure 2(b)). Despite relatively stable TLT-2 expression levels on peripheral blood-derived B cells, most B cell lines did not express TLT-2. Our results from both humans and mice [30,31] demonstrate that although TLT-2 expression is dominant on B cells and macrophages/monocytes, select conditioned T cells express substantial levels of TLT-2; moreover, continuous stimulation down-regulates rather than up-regulates TLT-2 expression.

\subsection{B7-H3 and TLT-2 Up-Regulates Cytokine Response of T Cells}

The functional roles of human B7-H3:TLT-2 pathway on $\mathrm{T}$ cell activation were examined. We previously reported marked enhancement of IL-2 production by stimulation of mTLT-2/DO11.10 cells with mB7-H3/P815 plus anti-CD3 mAb [30]. We also established a human version of the anti-CD3 mAb-induced costimulation assay system using P815 cells [30,34]. Human 4IgB7H3-transfected P815 (hB7-H3/P815) cells expressed markedly higher levels of B7-H3 than the parental P815 cells, but comparable levels of CD54 (ICAM-1) - a critical adhesion molecule - and Fc $\gamma \mathrm{R}$ (CD16/32) (Figure 3(a)). Human TLT-2/DO11.10 was stimulated with hB7$\mathrm{H} 3 / \mathrm{P} 815$ cells in the presence of anti-CD3 mAb. The subsequent increase in IL-2 production was dependent on hB7-H3/P815 cell density, which was efficiently inhibited by the addition of either one of 2 different antiTLT-2 mAbs (MIH59 and MIH60) (Figure 3(b), left). The anti-TLT-2 mAb (MIH60) inhibited IL-2 production from TLT-2/DO11.10 cells in a dose-dependent manner, whereas no inhibitory effects were observed in IL-2 production from DO11.10 cells lacking TLT-2 (Figure 3(b), right). These results suggest involvement of the TLT-2 pathway in B7-H3-induced IL-2 production. The role of the TLT- 2 pathway in peripheral blood $\mathrm{CD}^{+}$and $\mathrm{CD}^{+} \mathrm{T}$ cell activation was subsequently examined. Stimulation with hB7-H3/P815 cells slightly enhanced (a)

P815

hB7-H3/P815
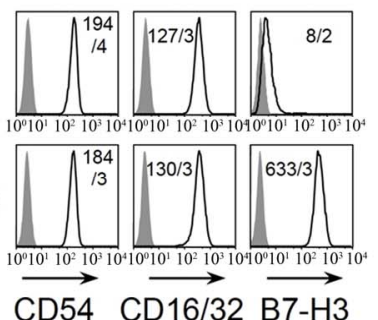

(b) $\mathrm{hTLT}-2 / \mathrm{DO} 11.102 \times 10^{4} /$ well hB7-H3/P815

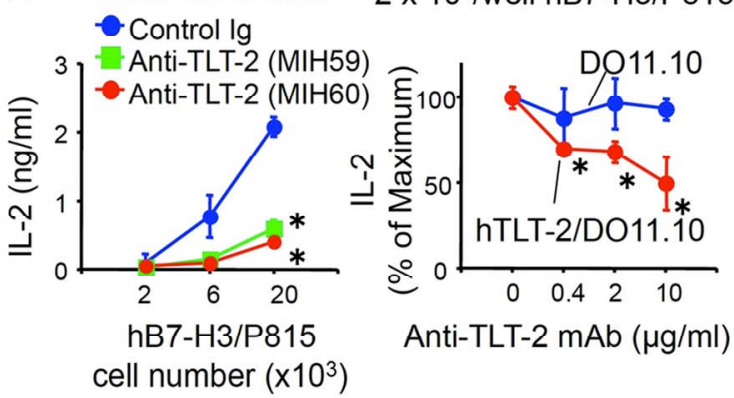

(c)

$\mathrm{CD} 4^{+}$

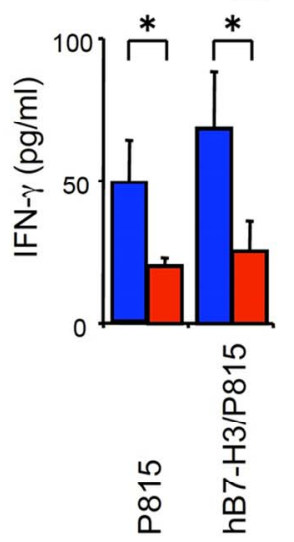

Control Ig

Anti-TLT-2

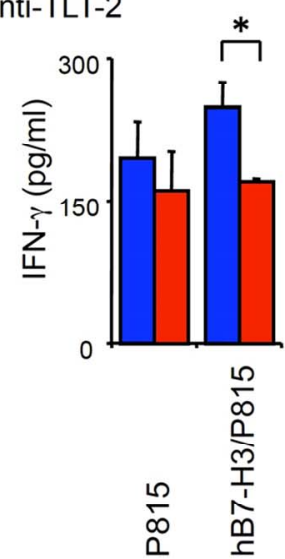

Figure 3. B7-H3:TLT-2 ligation up-regulates cytokine production from $\mathrm{T}$ cells. (a) The expression levels of CD54, $\mathrm{CD} 16 / 32$, and B7-H3 on parental or hB7-H3/P815 were examined with PE-anti-CD54, biotin-anti-CD16/32, or biotin-anti-mouse/human B7-H3 mAb (MIH32), followed by streptavidin-PE. Representative histogram profiles are shown with the control histograms nearest the ordinate (shaded); (b) hTLT-2/DO11.10 cells were stimulated with $1 \mu \mathrm{g} / \mathrm{ml} \mathrm{of}$ anti-CD3 mAb in the presence of hB7-H3/P815 at the indicated cell density in the presence of $10 \mu \mathrm{g} / \mathrm{ml}$ of control Ig or anti-TLT-2 mAb (MIH59 or MIH60) (left). Parental DO11.10 or hTLT-2/DO11.10 cells were stimulated with $1 \mu \mathrm{g} / \mathrm{ml}$ anti-CD3 mAb plus $2 \times 10^{4} /$ well hB7-H3/P815, in the presence of graded doses of anti-TLT-2 mAb (MIH60) (right). The result observed with $10 \mu \mathrm{g} / \mathrm{ml}$ of control $\mathrm{mAb}$ was similar to that observed in the absence of anti-TLT-2 $\mathrm{mAb}$; (c) $1 \times 10^{5}$ of $\mathrm{CD}^{+}$(left) or $\mathrm{CD}^{+}$(right) $\mathrm{T}$ cells peripheral blood from a healthy donor were stimulated with $1 \mu \mathrm{g} / \mathrm{ml}$ of anti-CD3 $\mathrm{mAb}$ plus $4 \times 10^{4}$ of parental P815 or hB7-H3/P815 in the presence of $10 \mu \mathrm{g} / \mathrm{ml}$ control Ig or anti-TLT-2 mAb (MIH60). IL-2 (b) and IFN- $\gamma$ (c) amounts in culture supernatants at days 1 and 4, respectively, were analyzed by ELISA. Data are represented as mean $\pm \mathrm{SD}$ values of triplicate cultures for IL-2 or IFN- $\gamma$ production ((b) left and (c)) or for percentage of maximal IL-2 production on each DO11.10 cell ((b) right). The data shown are representative of at least 2 independent experiments. ${ }^{*} p<0.05$, compared with control Ig ((b) left and (c)) and with anti-TLT-2 mAb-free cultures ((b) right). 
IFN- $\gamma$ production from both $\mathrm{CD}^{+}$and $\mathrm{CD} 8^{+} \mathrm{T}$ cells compared with parental P815 cell-stimulated cultures (Figure 3(c)). The addition of anti-TLT-2 mAb (MIH60) inhibited IFN- $\gamma$ production that was stimulated with either parental or hB7-H3/P815 cells, but inhibitory effects were preferentially evident in the culture with $\mathrm{hB} 7-\mathrm{H} 3 /$ P815. These results suggest that the TLT-2: B7-H3 pathway has a costimulatory function in both $\mathrm{CD}^{+}$and $\mathrm{CD} 8^{+} \mathrm{T}$ cell activation, as assessed by IFN- $\gamma$ production. Murine mastocytoma P815 cells express low levels of endogenous mouse 2IgB7-H3 [30]. Because both mouse 2IgB7-H3 and human 4IgB7-H3 could bind to human TLT-2 (Figure 1(c)), it is possible that interactions occurred between hTLT-2 and endogenous mB7-H3, which were efficiently inhibited by the addition of anti-hTLT-2 mAbs. Further studies are needed for elucidating this hypothesis. Our results thus suggest that the human TLT-2:B7-H3 pathway enhances T cell activation.

\section{DISCUSSION}

Similar to our previous observations in mice [30], we have demonstrated that a major form of human B7-H3 (4IgB7-H3) bound to human TLT-2, an interaction that costimulated $\mathrm{T}$ cell activation. Leitner et al. [8] reported that both forms of hB7-H3Ig (4Ig and 2Ig) did not interact with hTLT-2, and that $\mathrm{mB} 7-\mathrm{H} 3 \mathrm{Ig}$ did not bind to mTLT-2. At present, we cannot clearly explain the discrepancies in the results of the 2 studies. TLT- 2 expression levels on transfectants, methodologies of preparation and purification of fusion proteins, and binding detection methods by flow cytometry were different between the 2 studies. Notably, Leitner et al. performed the staining method using unlabeled B7-H3Ig, followed by PE-anti-human IgG [8]. This combination of staining reagents did not result in substantial positive binding in our hands. Our study utilized the system of biotinylated B7-H3Ig, followed by PE-streptavidin for amplifying the binding process. As Leitner et al. had observed using TLT-2Ig [20], we could not detect substantial binding when we used biotinylated mouse TLT-2Ig onto mouse B7-H3-transfectants. These results suggest that the binding capacity between B7-H3 and TLT-2 is very limited, and additional special conditions are required for visualization of TLT-2Ig binding to B7-H3 .

Consistent with our results in mice, the human TLT-2: B7-H3 pathway also showed costimulatory function in both $\mathrm{CD}^{+}$and $\mathrm{CD} 8^{+} \mathrm{T}$ cell activation. However, we suspect that the functional contribution of TLT-2:B7-H3 pathway to $\mathrm{T}$ cell activation in vivo is very limited, because TLT- 2 expression on T cells is tightly restricted, and $\mathrm{B} 7-\mathrm{H} 3$ expression on antigen-presenting cells is limited. The range of detection for costimulatory effects is very narrow, even under conditions of very high expression levels of B7-H3 and TLT-2 in vitro. Furthermore, in the experiments using peripheral blood $\mathrm{CD}^{+}$and $\mathrm{CD} 8^{+}$ $\mathrm{T}$ cells and $\mathrm{hB} 7-\mathrm{H} 3 / \mathrm{P} 815$ transfectants, a clear augmenttation in proliferative responses was not observed. This suggests a unique signaling mechanism via TLT-2 that is distinct from CD28- or ICOS (CD278)-mediated signaling. Indeed, the cytoplasmic domain of TLT-2 includes a potential SH3 domain-binding motif [28]. Src family molecules and Grb2, which have an SH3 domain, may play a role in downstream signaling. Further studies are needed for elucidation.

Our findings never rule out the B7-H3-mediated immune regulation that is often seen in patients with various tissue types of carcinomas. An initial report showed that human B7-H3Ig-binding protein was not detected on freshly isolated peripheral blood $\mathrm{T}$ cells, transiently induced at $24 \mathrm{~h}$ after PHA stimulation, and declined quickly at $48 \mathrm{~h}$ [4]. This expression profile differs slightly from the TLT-2 expression profile. In addition, we could not detect hTLT-2 on freshly isolated peripheral blood T cells using hB7-H3Ig, presumably because of the low affinity of the interaction. Another B7-H3-binding receptor other than TLT-2 may exist, and this putative receptor may induce co-inhibitory signals. In addition to membrane-bound B7-H3, soluble B7-H3 (sB7-H3) has been detected in sera from healthy donors [35] as well as patients with cancer $[36,37]$ and sepsis [38]. sB7-H3 has been shown to be released from monocytes, dendritic cells, and activated T cells [35]; moreover, a correlation between serum sB7-H3 levels and inflammatory cytokine levels (such as TNF- $\alpha$ and IL-6) has been observed in septic patients [38]. Release of $\mathrm{sB} 7-\mathrm{H} 3$ regulates the interactions of $\mathrm{B} 7-\mathrm{H} 3$ with its receptors. Presently, we have not examined whether $\mathrm{sB} 7-\mathrm{H} 3$ can bind cell surface TLT-2. It is possible that the secreted $\mathrm{sB} 7-\mathrm{H} 3$ binds TLT- 2 on macrophages and neutrophils, and modulates innate immune responses or interferes with the binding of the membrane form of B7-H3. Although the soluble form of TLT-2 has not yet been identified, soluble TREM1, TREM2, and TLT-1 receptor have been detected in sera from patients with infectious diseases and often correlates with disease severity [27]. If a soluble form of TLT-2 also exists, this may add further complexity to these signaling pathways. Although we only examined the role of TLT- 2 on T cells in this study, dominant expression of TLT-2 in macrophages suggests a dominant contribution of TLT- 2 to innate immune responses. This may affect cell migration and inflammatory responses, resulting in the modulation of adaptive immune responses. Contrasting roles of $\mathrm{B} 7-\mathrm{H} 3$ can probably be attributed to the existence of multiple receptors and soluble forms. In this study, we have demonstrated that human and mouse B7-H3 cross-interact with human and mouse TLT-2 species, and the interaction of B7-H3 with human TLT-2 additionally augments T cell responses. 


\section{ACKNOWLEDGEMENTS}

We thank T. Kitamura (the Institute of Medical Science, the University of Tokyo) for kindly providing retroviral vectors and the packaging cell line Plat-E; and S. Miyakoshi for cell sorting.

\section{REFERENCES}

[1] Greenwald, R.J., Freeman, G.J. and Sharpe, A.H. (2005) The B7 family revisited. Annual Review of Immunology, 23, 515-548. doi:10.1146/annurev.immunol.23.021704.115611

[2] Bour-Jordan, H., Esensten, J.H., Martinez-Llordella, M., Penaranda, C., Stumpf, M. and Bluestone, J.A. (2011) Intrinsic and extrinsic control of peripheral T-cell tolerance by costimulatory molecules of the CD28/B7 family. Immunological Reviews, 241, 180-205. doi:10.1111/j.1600-065X.2011.01011.X

[3] Zhu, Y., Yao, S. and Chen, L. (2011) Cell surface signaling molecules in the control of immune responses: A tide model. Immunity, 34, 466-478. doi:10.1016/j.immuni.2011.04.008

[4] Chapoval, A.I., Ni, J., Lau, J.S., Wilcox, R.A., Flies, D.B., Liu, D., Dong, H., Sica, G.L., Zhu, G., Tamada, K. and Chen, L. (2001) B7-H3: A costimulatory molecule for T cell activation and IFN- $\gamma$ production. Nature Immunology, 2, 269-274. doi:10.1038/85339

[5] Ling, V., Wu, P.W., Spaulding, V., Kieleczawa, J., Luxenberg, D., Carreno, B.M. and Collins, M. (2003) Duplication of primate and rodent $\mathrm{B} 7-\mathrm{H} 3$ immunoglobulin Vand C-like domains: Divergent history of functional redundancy and exon loss. Genomics, 82, 365-377. doi:10.1016/S0888-7543(03)00126-5

[6] Sun, M., Richards, S., Prasad, D.V., Mai, X.M., Rudensky, A. and Dong, C. (2002) Characterization of mouse and human B7-H3 genes. The Journal of Immunology, 168, 6294-6297.

[7] Zhou, Y.H., Chen, Y.J., Ma, Z.Y., Xu, L., Wang, Q., Zhang, G.B., Xie, F., Ge, Y., Wang, X.F. and Zhang, X.G. (2007) 4IgB7-H3 is the major isoform expressed on immunocytes as well as malignant cells. Tissue Antigens, 70, 96-104. doi:10.1111/j.1399-0039.2007.00853.x

[8] Leitner, J., Klauser, C., Pickl, W.F., Stockl, J., Majdic, O., Bardet, A.F., Kreil, D.P., Dong, C., Yamazaki, T., Zlabinger, G., Pfistershammer, K. and Steinberger, P. (2009) B7-H3 is a potent inhibitor of human T-cell activation: No evidence for B7-H3 and TREML2 interaction. European Journal of Immunology, 39, 1754-1764. doi:10.1002/eji.200839028

[9] Zhang, G.B., Dong, Q.M., Xu, Y., Yu, G.H. and Zhang, X.G. (2005) B7-H3: Another molecule marker for MoDCs? Cellular \& Molecular Immunology, 2, 307-311.

[10] Kim, J., Myers, A.C., Chen, L., Pardoll, D.M., TruongTran, Q.A., Lane, A.P., McDyer, J.F., Fortuno, L. and Schleimer, R.P. (2005) Constitutive and inducible expression of b7 family of ligands by human airway epithelial cells. American Journal of Respiratory Cell and Molecular Biology, 33, 280-289.

\section{doi:10.1165/rcmb.2004-01290C}

[11] Saatian, B., Yu, X.Y., Lane, A.P., Doyle, T., Casolaro, V. and Spannhake, E.W. (2004) Expression of genes for B7-H3 and other T cell ligands by nasal epithelial cells during differentiation and activation. American Journal of Physiology Lung Cell and Molecular Physiology, 287, L217-L225. doi:10.1152/ajplung.00132.2003

[12] Wu, C.P., Jiang, J.T., Tan, M., Zhu, Y.B., Ji, M., Xu, K.F., Zhao, J.M., Zhang, G.B. and Zhang, X.G. (2006) Relationship between co-stimulatory molecule B7-H3 expression and gastric carcinoma histology and prognosis. World Journal of Gastroenterology, 12, 457-459.

[13] Sun, Y., Wang, Y., Zhao, J., Gu, M., Giscombe, R., Lefvert, A.K. and Wang, X. (2006) B7-H3 and B7-H4 expression in non-small-cell lung cancer. Lung Cancer, 53, 143-151. doi:10.1016/j.lungcan.2006.05.012

[14] Roth, T.J., Sheinin, Y., Lohse, C.M., Kuntz, S.M., Frigola, X., Inman, B.A., Krambeck, A.E., McKenney, M.E., Karnes, R.J., Blute, M.L., Cheville, J.C., Sebo, T.J. and Kwon, E.D. (2007) B7-H3 ligand expression by prostate cancer: A novel marker of prognosis and potential target for therapy. Cancer Research, 67, 7893-7900. doi:10.1158/0008-5472.CAN-07-1068

[15] Castriconi, R., Dondero, A., Augugliaro, R., Cantoni, C., Carnemolla, B., Sementa, A.R., Negri, F., Conte, R., Corrias, M.V., Moretta, L., Moretta, A. and Bottino, C. (2004) Identification of 4Ig-B7-H3 as a neuroblastoma-associated molecule that exerts a protective role from an NK cellmediated lysis. Proceedings of the National Academy of Sciences USA, 101, 12640-12645.

doi:10.1073/pnas.0405025101

[16] Chen, Y.W., Tekle, C. and Fodstad, O. (2008) The immunoregulatory protein human $\mathrm{B} 7 \mathrm{H} 3$ is a tumor-associated antigen that regulates tumor cell migration and invasion. Curr. Cancer Drug Targets, 8, 404-413. doi: $10.2174 / 156800908785133141$

[17] Tran, C.N., Thacker, S.G., Louie, D.M., Oliver, J., White, P.T., Endres, J.L., Urquhart, A.G., Chung, K.C. and Fox, D.A. (2008) Interactions of T cells with fibroblast-like synoviocytes: Role of the B7 family costimulatory ligand B7-H3. Journal of Immunology, 180, 2989-2998.

[18] Waschbisch, A., Wintterle, S., Lochmuller, H., Walter, M.C., Wischhusen, J., Kieseier, B.C. and Wiendl, H. (2008) Human muscle cells express the costimulatory molecule B7-H3, which modulates muscle-immune interactions. Arthritis \& Rheumatism, 58, 3600-3608. doi:10.1002/art.23997

[19] Yang, H.Y., Chu, M., Zheng, L.W., Zwahlen, R.A., Luo, J., Zou, D.H. and Sun, S.T. (2008) Transgenic B7-H3 therapy induces tumor-specific immune response in human oral squamous cell cancer: an in vitro study. Oral Surgery, Oral Medicine, Oral Pathology, Oral Radiology \& Endod, 106, 721-728. doi:10.1016/j.tripleo.2008.08.012

[20] Zang, X., Thompson, R.H., Al-Ahmadie, H.A., Serio, A.M., Reuter, V.E., Eastham, J.A., Scardino, P.T., Sharma, P. and Allison, J.P. (2007) B7-H3 and B7x are highly expressed in human prostate cancer and associated with disease spread and poor outcome. Proceedings of the $\mathrm{Na}$ - 
tional Academy of Sciences USA, 104, 19458-19463. doi:10.1073/pnas.0709802104

[21] Crispen, P.L., Sheinin, Y., Roth, T.J., Lohse, C.M., Kuntz, S.M., Frigola, X., Thompson, R.H., Boorjian, S.A., Dong, H., Leibovich, B.C., Blute, M.L. and Kwon, E.D. (2008) Tumor cell and tumor vasculature expression of B7-H3 predict survival in clear cell renal cell carcinoma. Clinical Cancer Research, 14, 5150-5157. doi:10.1158/1078-0432.CCR-08-0536

[22] Katayama, A., Takahara, M., Kishibe, K., Nagato, T., Kunibe, I., Katada, A., Hayashi, T. and Harabuchi, Y. (2011) Expression of B7-H3 in hypopharyngeal squamous cell carcinoma as a predictive indicator for tumor metastasis and prognosis. International Journal of Oncology, 38, 1219-1226. doi:10.3892/ijo.2011.949

[23] Sun, J., Chen, L.J., Zhang, G.B., Jiang, J.T., Zhu, M., Tan, Y., Wang, H.T., Lu, B.F. and Zhang, X.G. (2011) Clinical significance and regulation of the costimulatory molecule B7-H3 in human colorectal carcinoma. Cancer Immunology, Immunotherapy, 59, 1163-1171. doi:10.1007/s00262-010-0841-1

[24] Yamato, I., Sho, M., Nomi, T., Akahori, T., Shimada, K., Hotta, K., Kanehiro, H., Konishi, N., Yagita, H. and Nakajima, Y. (2009) Clinical importance of B7-H3 expression in human pancreatic cancer. British Journal of Cancer, 101, 1709-1716. doi:10.1038/sj.bjc.6605375

[25] Loos, M., Hedderich, D.M., Ottenhausen, M., Giese, N.A., Laschinger, M., Esposito, I., Kleeff, J. and Friess, H. (2009) Expression of the costimulatory molecule B7-H3 is associated with prolonged survival in human pancreatic cancer. BMC Cancer, 9, 463. doi:10.1186/1471-2407-9-463

[26] Klesney-Tait, J., Turnbull, I.R. and Colonna, M. (2006) The TREM receptor family and signal integration. Nature Immunology, 7, 1266-1273. doi:10.1038/ni1411

[27] Ford, J.W. and McVicar, D.W. (2009) TREM and TREMlike receptors in inflammation and disease. Current Opinion in Immunology, 21, 38-46. doi:10.1016/j.coi.2009.01.009

[28] King, R.G., Herrin, B.R. and Justement, L.B. (2006) Tremlike transcript 2 is expressed on cells of the myeloid/ granuloid and B lymphoid lineage and is up-regulated in response to inflammation. Journal of Immunology, 176, 6012-6021.

[29] Halpert, M.M., Thomas, K.A., King, R.G. and Justement, L.B. (2011) TLT2 potentiates neutrophil antibacterial activity and chemotaxis in response to $G$ protein-coupled receptor-mediated signaling. Journal of Immunology, 187, 2346-2355. doi:10.4049/jimmunol.1100534
[30] Hashiguchi, M., Kobori, H., Ritprajak, P., Kamimura, Y., Kozono, H. and Azuma, M. (2008) Triggering receptor expressed on myeloid cell-like transcript 2 (TLT-2) is a counter-receptor for B7-H3 and enhances T cell responses. Proceedings of the National Academy of Sciences USA, 105, 10495-10500. doi:10.1073/pnas.0802423105

[31] Kobori, H., Hashiguchi, M., Piao, J., Kato, M., Ritprajak, P. and Azuma, M. (2010) Enhancement of effector CD8+ T-cell function by tumour-associated B7-H3 and modulation of its counter-receptor triggering receptor expressed on myeloid cell-like transcript 2 at tumour sites. Immunology, 130, 363-373. doi:10.1111/j.1365-2567.2009.03236.x

[32] Otsuki, N., Kamimura, Y., Hashiguchi, M. and Azuma, M. (2006) Expression and function of the B and T lymphocyte attenuator (BTLA/CD272) on human T cells. Biochemical and Biophysical Research Communications, 344, 1121-1127. doi:10.1016/i.bbrc.2006.03.242

[33] Youngnak, P., Kozono, Y., Kozono, H., Iwai, H., Otsuki, N., Jin, H., Omura, K., Yagita, H., Pardoll, D.M., Chen, L. and Azuma, M. (2003) Differential binding properties of B7-H1 and B7-DC to programmed death-1. Biochemical and Biophysical Research Communications, 307, 672-677. doi:10.1016/S0006-291X(03)01257-9

[34] Azuma, M., Cayabyab, M., Buck, D., Phillips, J.H. and Lanier, L.L. (1992) CD28 interaction with B7 costimulates primary allogeneic proliferative responses and cytotoxicity mediated by small, resting $\mathrm{T}$ lymphocytes. The Journal of Experimental Medicine, 175, 353-360. doi:10.1084/jem.175.2.353

[35] Zhang, G., Hou, J., Shi, J., Yu, G., Lu, B. and Zhang, X. (2008) Soluble CD276 (B7-H3) is released from monocytes, dendritic cells and activated $\mathrm{T}$ cells and is detectable in normal human serum. Immunology, 123, 538-546. doi:10.1111/j.1365-2567.2007.02723.x

[36] Sun, J., Chen, L.J., Zhang, G.B., Jiang, J.T., Zhu, M., Tan, Y., Wang, H.T., Lu, B.F. and Zhang, X.G. (2010) Clinical significance and regulation of the costimulatory molecule B7-H3 in human colorectal carcinoma. Cancer Immunology, Immunotherapy, 59, 1163-1171. doi:10.1007/s00262-010-0841-1

[37] Zhang, G., Xu, Y., Lu, X., Huang, H., Zhou, Y., Lu, B. and Zhang, X. (2009) Diagnosis value of serum B7-H3 expression in non-small cell lung cancer. Lung Cancer, 66, 245-249. doi:10.1016/j.lungcan.2009.01.017

[38] Zhang, G., Wang, J., Kelly, J., Gu, G., Hou, J., Zhou, Y., Redmond, H.P., Wang, J.H. and Zhang, X. (2010) B7-H3 augments the inflammatory response and is associated with human sepsis. Journal of Immunology, 185, 36773684. doi:10.4049/jimmunol.0904020 